Carrier transport properties in the vicinity of single self-assembled quantum dots determined by low-voltage cathodoluminescence imaging

E. Dupuy, D. Morris, N. Pauc, V. Aimez, M. Gendry, and D. Drouin

Citation: Appl. Phys. Lett. 94, 022113 (2009); doi: 10.1063/1.3072613

View online: http://dx.doi.org/10.1063/1.3072613

View Table of Contents: http://aip.scitation.org/toc/apl/94/2

Published by the American Institute of Physics

A.P|Applied Physics Letters

If your article has the

Save your money for your research.

It's now FREE to publish with us -

no page, color or publication charges apply. 


\title{
Carrier transport properties in the vicinity of single self-assembled quantum dots determined by low-voltage cathodoluminescence imaging
}

\author{
E. Dupuy, ${ }^{1,3}$ D. Morris, ${ }^{1}$ N. Pauc, ${ }^{2, a)}$ V. Aimez, ${ }^{2}$ M. Gendry, ${ }^{3}$ and D. Drouin ${ }^{2, b)}$ \\ ${ }^{1}$ CNR2, Département de Physique, Université de Sherbrooke, Sherbrooke, Quebec J1K2R1, Canada \\ ${ }^{2}$ CNR2, Département de Génie Électrique et Génie Informatique, Université de Sherbrooke, Sherbrooke, \\ Québec J1K2R1, Canada \\ ${ }_{3}^{3}$ Institut des Nanotechnologies de Lyon, Ecole Centrale de Lyon, INL-UMR5270/CNRS, 69134 Ecully, \\ France
}

(Received 19 November 2008; accepted 23 December 2008; published online 16 January 2009)

\begin{abstract}
We propose a method to investigate the carrier transport properties in the ultrathin wetting layer of a self-assembled quantum dot (QD) structure using low-voltage cathodoluminescence (CL) imaging. Measurements are performed on diluted InAs/InP QDs in order to spatially resolve them on CL images at temperature ranging from 5 to $300 \mathrm{~K}$. The mean ambipolar diffusion length extracted from CL intensity profiles across different isolated bright spots is about $300 \mathrm{~nm}$ at $300 \mathrm{~K}$. This gives an ambipolar carrier mobility of about $110 \mathrm{~cm}^{2} /(\mathrm{V} \mathrm{s})$. Temperature investigation reveals a maximum diffusion length near 120 K. () 2009 American Institute of Physics.
\end{abstract}

[DOI: $10.1063 / 1.3072613]$

With the development of solid-state optoelectronic devices based on single self-assembled quantum dots (QDs) such as nanolasers or single photon sources, evaluation of their individual properties is fundamental. As well as the study of single dot emission, investigation of carrier transport from their generation point in the barrier to their capture and relaxation into QDs is relevant for the improvement of device performances. ${ }^{1}$ Time-related properties of carrier transport such as carrier capture time and relaxation time of single QDs have been studied by a few ultrafast and spatially resolved spectroscopy techniques. ${ }^{2-4}$ To determine the carrier diffusion length before QD capture, such techniques require knowing the local carrier diffusion constant or mobility in the material. A direct measurement of the carrier diffusion length is thus attractive. Various techniques have been used to directly determine this property in low dimensional semiconductor structures. ${ }^{5-10}$ However, for self-assembled QD structures, the exciting probe size in most cases is larger than the distance between each dot preventing from any measurement in the vicinity of a single QD.

Here, we propose a low-voltage cathodoluminescence (CL) method to directly measure the excess carrier diffusion length in the wetting layer (WL) before capture into one specific QD. Interest in such technique is driven by a high lateral spatial resolution, its contactless character, and its ease of use without any special sample preparation such as microfabrication of mesas or metal apertures to isolate a single QD. The experimental CL spatial resolution is generally limited by the distance $d_{\mathrm{CL}}=\sqrt{\phi_{p}^{2}+R_{e}^{2}+L_{d}^{2}}$, where $\phi_{p}$ is the electron beam probe diameter, $R_{e}$ is the radius of the in-plane primary electron interaction volume, and $L_{d}$ is the diffusion length of the generated carriers. ${ }^{11}$ Using a lowvoltage CL probe beam $(1 \mathrm{kV})$ and a field emission electron source, we can reduce $\phi_{p}$ and $R_{e}$ toward a quasipoint source

\footnotetext{
${ }^{\text {a) }}$ Present address: CEA/Grenoble-INAC/SP2M/SiNaPS, 17 rue des Martyrs, 38054 Grenoble Cedex 9, France.

${ }^{\text {b) }}$ Author to whom correspondence should be addressed. Electronic mail: dominique.drouin@usherbrooke.ca.
}

in comparison with the diffusion length in III-V bulk semiconductors. Under these conditions, if the CL intensity comes only from one QD's emission, the limited resolution distance becomes the carrier diffusion length before capture into one specific dot.

Figure 1 shows the schematic of the experiment. A lowvoltage electron beam generates excess carriers in the barrier layer just below the surface of the QD structure. Most of them relax very rapidly into the WL in the picosecond range, ${ }^{12}$ then diffuse through it, ${ }^{13}$ and finally get captured into the QDs where they can recombine and emit light. Other carriers are either captured by surface defects near the beam impact or recombine in the barrier and WL. So, we can assume that carrier diffusion takes place mainly in the WL when the scanning electron beam is far from the dot. Furthermore, the density of electron-hole pairs generated in this confined region close to the surface is very high and much greater than the barrier majority carrier concentration. Thus, only ambipolar diffusion lengths of excess carriers in the WL can be determined. The diffusion length is deduced from CL intensity profile $I(R)$ over the QD with $R$ as the distance between the probe and the QD.

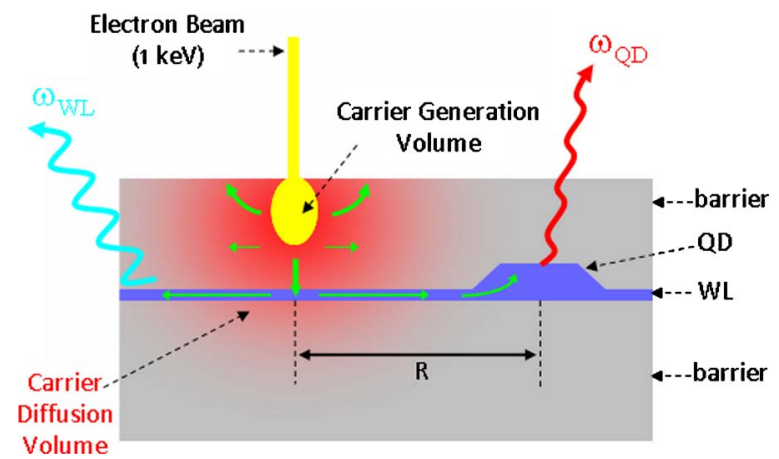

FIG. 1. (Color online) Schematic of the experiment. Excess carriers generated in sub-10-nm volume of the barrier are mainly captured by the WL and diffuse through it before their final capture into a QD. Diffusion length is deduced from CL intensity profile $I(R)$ over the QD. 


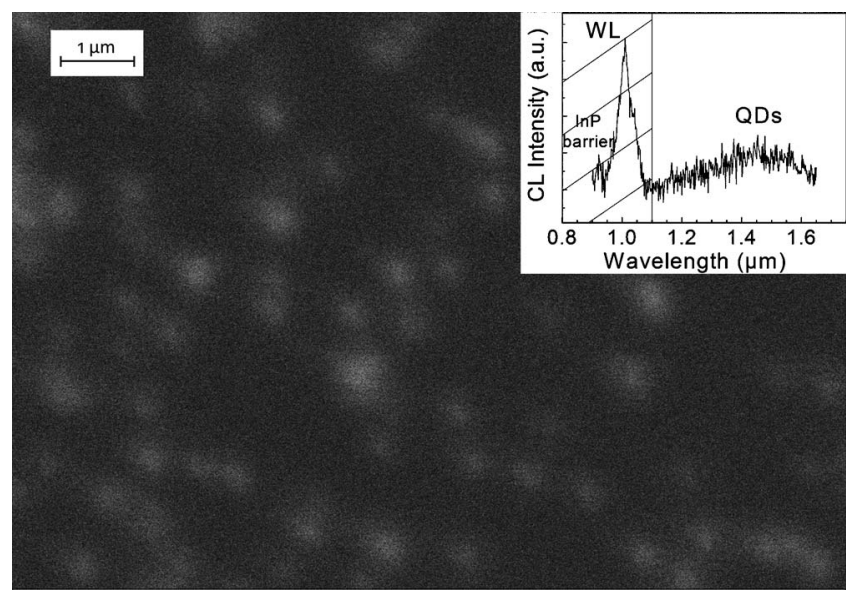

FIG. 2. CL intensity micrograph of a low QD density InAs/InP sample, acquired at $1 \mathrm{kV}$ and $300 \mathrm{~K}$ using a long pass filter cutoff at $1.1 \mu \mathrm{m}$. The luminescence on the map corresponds to the QDs emitting between 1.1 and $1.65 \mu \mathrm{m}$ as shown by the CL spectrum (inset).

The investigated structure is made of InAs selfassembled QDs grown by solid-source molecular beam epitaxy on a semi-insulating $\operatorname{InP}(001)$ substrate. ${ }^{14}$ In order to spatially resolve QD luminescence, a specific growth procedure was implemented to produce diluted QDs, ${ }^{15}$ whose density is reduced to about $1 \mathrm{QD} / \mu \mathrm{m}^{2}$. The resulting dots are truncated pyramids with a typical lateral size of 30-40 nm and height of 2-5 nm. The InAs WL below the QDs has a mean thickness of 2 ML. QDs are capped with a thin $20 \mathrm{~nm}$ InP layer to allow excess carriers generated near the surface to reach the WL region. CL measurements were performed in a Zeiss Supra 55 VP field emission scanning electron microscope equipped with a continuous helium flow cryostat providing temperature from 5 to $300 \mathrm{~K}$. The luminescence was dispersed by a $30 \mathrm{~cm}$ grating monochromator and detected by a cooled InGaAs cathode photomultiplier tube within the $0.3-1.65 \mu \mathrm{m}$ spectral range. CL micrographs were performed using a long pass cutoff filter at $1.1 \mu \mathrm{m}$ and a $1 \mathrm{kV}$ focused electron beam at several probe currents $\left(I_{b}\right)$. At $1 \mathrm{kV}, \phi_{p}$ is about $3 \mathrm{~nm}$ and according to Monte Carlo simulations, ${ }^{16}$ electron-hole pairs are injected into sub-10-nm volumes in the InP barrier providing a quasipoint source of carriers.

Figure 2 shows a CL image of such an array of InAs/InP QDs obtained at $1 \mathrm{kV}, 300 \mathrm{~K}$, and for $I_{b}=3 \mathrm{nA}$. The bright spots are correlated with the emission of individual dots. This has been confirmed by observing complementary features on WL and QD CL images of the same area at low temperatures and by comparing the density of bright spots with QD density estimated by AFM measurements on an uncapped sample. The measured spectrum of the same sample is presented (inset). We can clearly see the WL emission around $1 \mu \mathrm{m}$ and the QD emission starting at $1.1 \mu \mathrm{m}$ and extending up to $1.65 \mu \mathrm{m}$. The dashed area on the image represents the wavelength range removed using the optical filter.

Figure 3(a) shows the CL image over a specific region obtained at higher magnification and its related CL intensity profile for $I_{b}=3 \mathrm{nA}$. When the electron beam is scanned above one QD, the CL signal is nearly constant since carriers are captured efficiently into this dot. For an excitation spot away from the dot, the CL signal decreases since part of the
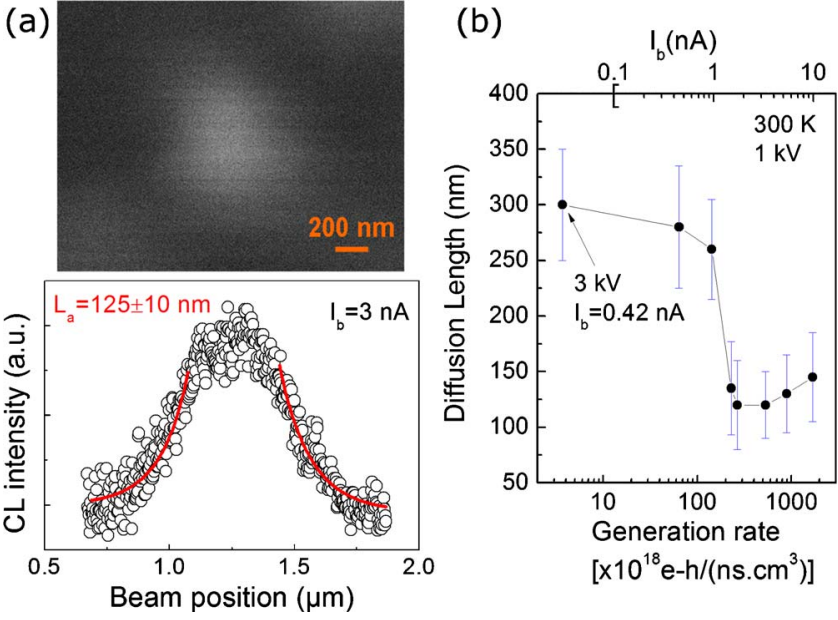

FIG. 3. (Color online) (a) Zoom on a submicrometer bright spot associated with a single QD and its $\mathrm{CL}$ intensity profile at $300 \mathrm{~K}$ for $I_{b}=3 \mathrm{nA}$. The ambipolar diffusion length $L_{a}$ is deduced from the $\operatorname{csch}(R / L)$ fitting curves (solid line). (b) $L_{a}$ values as a function of beam intensity and carrier generation rate at $1 \mathrm{kV}$ and $300 \mathrm{~K}$. One measure is made at $3 \mathrm{kV}$ and $0.42 \mathrm{nA}$ as indicated. Error bars correspond to the calculated mean standard deviation.

excess carriers recombine in the WL. For ambipolar diffusion of carriers, the local density of electrons and holes is obtained by solving the continuity equation under a steady state regime. If we assume that dots act as perfect sinks, the diffusion current will be more pronounced toward the nearest dot direction. A one-dimensional diffusion equation can then be used to obtain the approximate distribution of carriers from the generated point at $r=0$ to the dot position at $r=R$. This is a well known diffusion problem with trivial limit conditions at $r=0$, diffusion current equals generation rate, and at $r=R$, carrier density in the WL is zero. The carrier diffusion current evaluated at the dot position is given by $I(R) \propto \operatorname{csch}\left(R / L_{a}\right)$, where $L_{a}$ is the ambipolar diffusion length. This expression is valid for $R \gg \phi_{p}, \mathrm{Re}, r_{0}$, the QD radius. We assume that the QD CL signal is linearly proportional to this diffusion current at $r=R$. The mean $L_{a}$ value is then obtained through the fit of $15 \mathrm{CL}$ intensity profiles across isolated bright spots taken randomly at different sample areas. Figure 3(b) presents mean carrier diffusion length values as a function of probe current and carrier generation rate at $300 \mathrm{~K}$. For the lowest carrier injection at $3 \mathrm{kV}$ and $I_{b}=0,42 \mathrm{nA}$, we found $300 \pm 50 \mathrm{~nm}$, which is in accordance with another group value ${ }^{17}$ taken at low-level injection. It decreases down to $120 \pm 30 \mathrm{~nm}$ and then slightly increases at higher injections. Enhanced nonradiative carrier recombination by Auger processes in the WL is suggested as an explanation for the reduced diffusion length observed at higher excitation current. The slight increase might be attributed to enhanced carrier diffusion into the InP barrier as the WL state filling becomes more important. One can note that the calculated standard deviation $\left(\Delta L_{a}=50 \mathrm{~nm}\right)$ is much larger than the mean deviation in the lateral size of our QD ensemble (about $15 \mathrm{~nm}$ ). This high $\Delta L_{a}$ value suggests that there exist local carrier transport inhomogeneities in the WL, most probably resulting from interface roughness, InAs $\mathrm{P}_{(1-x)}$ alloy composition and strain induced QD size effects. Ambipolar diffusion length is related to the carrier mobility by this $L_{a}=\sqrt{\mu \tau k_{B} T / e}$ expression, where $\tau$ is the carrier lifetime in a WL in the absence of QDs (recombina- 


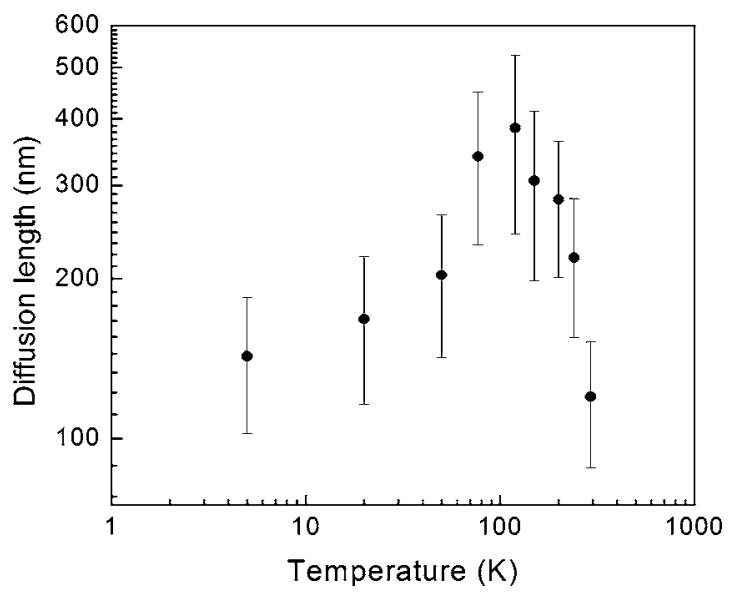

FIG. 4. Temperature dependence of carrier diffusion length before QD capture for $I_{b}=3 \mathrm{nA}$. Error bars correspond to the mean standard deviation.

tion in the dot is taken into account in the boundary condition). From low-excitation density time-resolved photoluminescence measurements, Raymond et al. ${ }^{18}$ determined a $\tau$ value of about $330 \mathrm{ps}$ in an ultrathin InAs quantum well, comparable in thickness to our WL. If we consider this $\tau$ value for the $300 \mathrm{~nm} L_{a}$ measurement obtained at $300 \mathrm{~K}$, we obtain an ambipolar mobility value of about $110 \pm 10 \mathrm{~cm}^{2} /(\mathrm{V} \mathrm{s})$. By assuming the same carrier mobility as we increase the current to $3 \mathrm{nA}$, we obtain from the measured $L_{a}$ value a limited Auger recombination lifetime of about 50 ps.

Mean carrier diffusion lengths have also been measured between 5 and $300 \mathrm{~K}$ for $I_{b}=3 \mathrm{nA}$ (Fig. 4). The $L_{a}$ value increases from 120 to $390 \mathrm{~nm}$ between 300 and $120 \mathrm{~K}$ and then decreases down to $140 \mathrm{~nm}$ at $5 \mathrm{~K}$. The carrier mobility also peaks around $100 \mathrm{~K}$ in III-V semiconductor quantum wells. ${ }^{19,20}$ Moreover, the carrier lifetime in a thin quantum well is expected to be nearly constant at low temperatures $(T<120 \mathrm{~K})$ and to decrease at higher temperatures due to thermal activation of the carriers and recombination into the InP barrier. We thus obtain a fair qualitative agreement with the expected $L_{a}(T)$ function. It is important to emphasize the fact that the diffusion length increases by only a factor of 3 between 5 and $100 \mathrm{~K}$, whereas an enhancement of 42 $\approx(100 / 5)^{5 / 4}$ is expected if carrier scattering by ionized impurities limits the mobility in this temperature range. Our results indicate that interface roughnesses and alloy composition fluctuations play a key role in limiting the carrier mobility in an ultrathin WL

In conclusion, we investigated the transport properties of excess carriers in the WL of a diluted InAs QD structure using low-voltage CL imaging with submicron spatial reso- lution. This method provides a direct measurement of the carrier diffusion length in the WL. An ambipolar carrier mobility of about $110 \mathrm{~cm}^{2} /(\mathrm{V} \mathrm{s})$ is estimated from the $300 \mathrm{~K}$ $L_{a}$ value. The $T$-dependence of the diffusion length is difficult to model but our results give valuable insights on possible scattering mechanisms in this InAs/InP system.

This work was partly supported by Canadian grant funds (NSERC, FQRNT, and Nano-Québec) and by the program Frontenac, which supports France/Québec exchange graduate students. E.D. would like to thank Jean Beerens, Pierre Lafrance, and Marcel Zakorzermy for their technical support.

${ }^{1}$ J. Wang, U. A. Griesinger, and H. Schweizer, Appl. Phys. Lett. 69, 1585 (1996).

${ }^{2}$ M. Ono, K. Matsuda, T. Saiki, K. Nishi, T. Mukaiyama, and M. KuwataGonokami, Jpn. J. Appl. Phys., Part 2 38, L1460 (1999).

${ }^{3}$ V. Zwiller, M. Pistol, D. Hessman, R. Coderstrom, W. Seifert, and L. Samuelson, Phys. Rev. B 59, 5021 (1999).

${ }^{4}$ M. Merano, S. Sonderegger, A. Crottini, S. Collin, P. Renucci, E. Pelucchi, A. Malko, M. H. Baier, E. Kapon, B. Deveaud, and J. D. Ganiere, Nature (London) 438, 479 (2005).

${ }^{5}$ A. Gustafsson, M. E. Pistol, L. Montelius, and L. Samuelson, J. Appl. Phys. 84, 1715 (1998), and references therein.

${ }^{6}$ H. A. Zarem, P. C. Sercel, J. A. Lebens, L. E. Eng, A. Yariv, and K. J. Vahala, Appl. Phys. Lett. 55, 1647 (1989).

${ }^{7}$ N. M. Haegel, J. D. Fabbri, and M. P. Coleman, Appl. Phys. Lett. 84, 1329 (2004)

${ }^{8}$ A. Fiore, M. Rossetti, B. Alloing, C. Paranthoen, J. X. Chen, L. Geelhaar, and H. Riechert, Phys. Rev. B 70, 205311 (2004).

${ }^{9}$ D. P. Popescu, P. G. Eliseev, A. Stintz, and K. J. Malloy, J. Appl. Phys. 94, 2454 (2003).

${ }^{10}$ A. F. G. Monte, F. V. de Sales, S. W. da Silva, M. A. G. Soler, J. M. R. Cruz, P. C. Morais, M. J. da Silva, A. A. Quivy, and J. R. Leite, Physica E (Amsterdam) 17, 122 (2003).

${ }^{11}$ V. I. Petrov, Bull. Acad. Sci. USSR, Phys. Ser. (Engl. Transl.) 56, 287 (1992).

${ }^{12}$ J. Siegert, S. Marcinkevičius, and Q. X. Zhao, Phys. Rev. B 72, 085316 (2005).

${ }^{13}$ D. Araújo, G. Olegart, J.-D. Ganière, and F.-K. Reinhart, J. Appl. Phys. 76, 342 (1994).

${ }^{14}$ E. Dupuy, P. Regreny, Y. Robach, M. Gendry, N. Chauvin, E. Tranvouez, G. Bremond, C. Bru-Chevallier, and G. Patriarche, Appl. Phys. Lett. 89, 123112 (2006).

${ }^{15}$ E. Dupuy, Proceedings of the 20th International Conference on Indium Phosphide and Related Materials, Versailles, France, 25-29 May 2008 (unpublished).

${ }^{16}$ For the simulations of the primary electron trajectories in solids, we used the CASINO software: http://www.gel.usherb.ca/casino. For more information, see D. Drouin, A. Real-Couture, D. Joly, X. Tastet, V. Aimez, and R. Gauvin, Scanning 29, 92 (2007)

${ }^{17}$ A. Gustafsson, D. Hessman, L. Samuelson, J. F. Carlin, R. Houdré, and A. Rudra, J. Cryst. Growth 147, 27 (1995).

${ }^{18}$ S. Raymond, K. Hinzer, S. Fafard, and J. L. Merz, Phys. Rev. B 61, R16331 (2000).

${ }^{19}$ H. Hillmer, A. Forchel, S. Hansmann, M. Morohashi, E. Lopez, H. P. Meier, and K. Ploog, Phys. Rev. B 39, 10901 (1989).

${ }^{20}$ L.-L. Chao, G. S. Cargill III, E. Snoeks, T. Marshall, J. Petruzzello, and M. Pashley, Appl. Phys. Lett. 74, 741 (1999). 